\title{
An Original Miniature Automated Ultrasound-Based System for Spinal Anaesthesia Against Usual Sonography: Preliminary Case-Serie
}

Van Hecke D., MD, Pandin P., MD, Naik H., MD, Estruch I.,MD, Van Obbergh L. MD, PhD, Dept of Anaesthesiology \& Intensive Care - CUB ERASMUS HOSPITAL,

Université Libre de Bruxelles, Belgium

\section{Background and Goal of study}

$\checkmark$ Blinded spinal anaesthesia (SA) remains a gold standard.

$\checkmark$ Preliminary ultrasound (US) scan may improve safety and efficacy ${ }^{1}$.

$\checkmark$ Few anaesthetists currently perform it (lack of understanding ${ }^{1}+$ poor US imaging ${ }^{2}$ )

$\checkmark$ A miniature automated ultrasound-based system (MAUBS), as anatomical structure tracker, might be a solution for Dura mater (D) location during SA. The MAUBS (Accuro ${ }^{\mathrm{TM}}$, Rivanna ${ }^{\mathrm{TM}}$ ) principle is to detect first the facet joins in the US field, measure their depth and then, fuse (SpineNav3D ${ }^{\mathrm{TM}}$ technology) with a 3D spine anatomical model for real-time 3D lumbar navigation to facilitate needle insertion.

$\checkmark$ We report a preliminary case-series comparing MAUBS data against BW 2D S and depth measurement on the needle.

\section{Methods (figure 1)}

$\checkmark$ Ten patients (orthopaedic surgery) were included (2.5ml HB bupi $0.5 \%+$ Suf $5 \mu$ g SA with 90 vs $150 \mathrm{~mm}$ long $25 \mathrm{G}$ Witacre needle).

$\checkmark$ Figure 1 displays the 4 steps methodology regarding the multimodal D depth assessment (steps 1-3) and verification on SA needle (step 4).

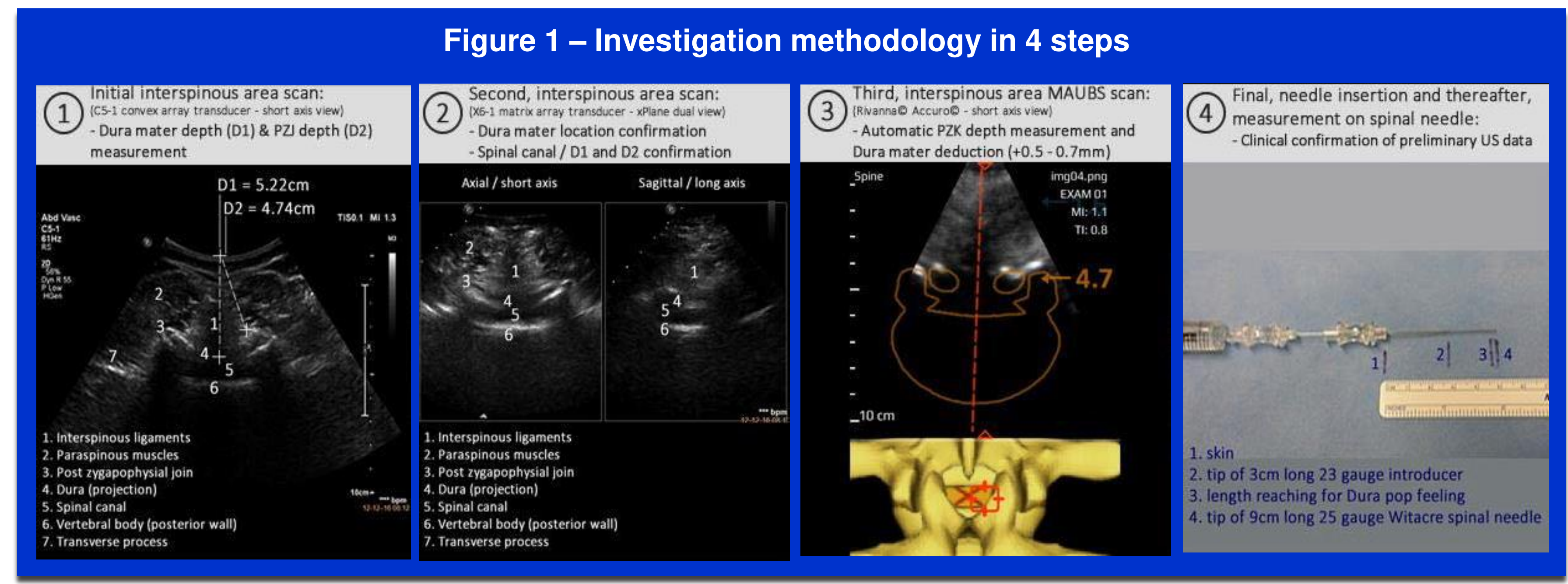

Results

Table 1 - Demographics and results

\begin{tabular}{|c|c|c|c|}
\hline Patient & $\begin{array}{c}\text { Body Mass Index } \\
\left(\mathbf{k g} / \mathbf{m}^{2}\right)\end{array}$ & Years old & $\begin{array}{c}\text { Time duration (sec) } \\
\text { step 1/2/3/4 }\end{array}$ \\
\hline 1 & 23,8 & 26 & $170 / 120 / 105 / 125$ \\
\hline 2 & 24 & 56 & $155 / 110 / 105 / 145$ \\
\hline 3 & 50,8 & 35 & $175 / 150 / 155 / 160$ \\
\hline 4 & 44,6 & 53 & $172 / 126 / 174 / 172$ \\
\hline 5 & 23,8 & 83 & $187 / 111 / 128 / 188$ \\
\hline 6 & 24,4 & 82 & $179 / 96 / 195 / 220$ \\
\hline 7 & 21 & 23 & $146 / 144 / 100 / 167$ \\
\hline 8 & 34 & 60 & $186 / 121 / 144 / 212$ \\
\hline 9 & 27,5 & 52 & $177 / 158 / 151 / 174$ \\
\hline 10 & 28,3 & 42 & $167 / 134 / 169 / 184$ \\
\hline
\end{tabular}

Figure 2 - BW 2D US vs MAUBS vs needle respective depths

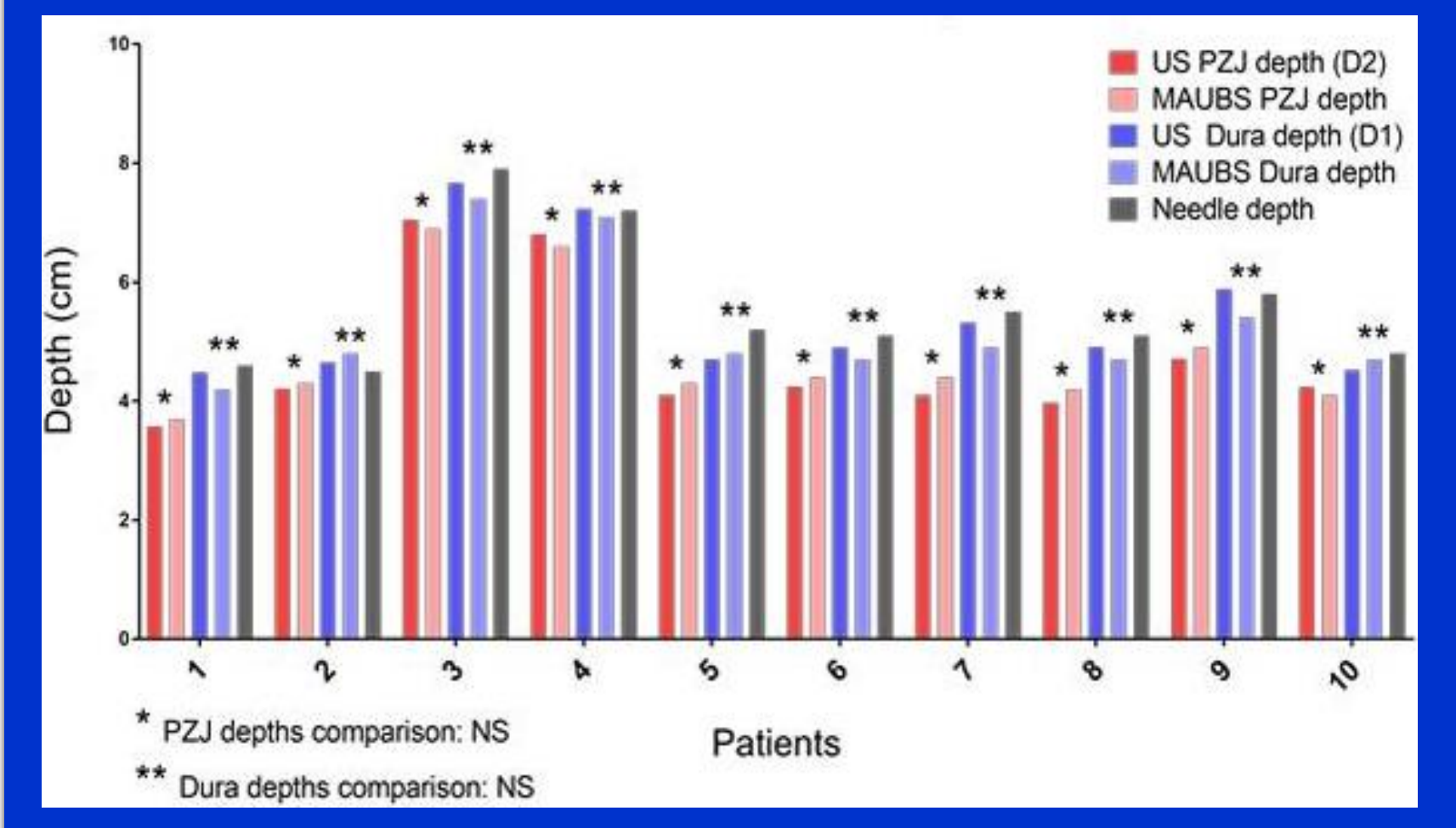

\section{Conclusions}

$\checkmark$ These present conclusive results encourage the study of the MAUBS learning curve and its impact on SA practice. 